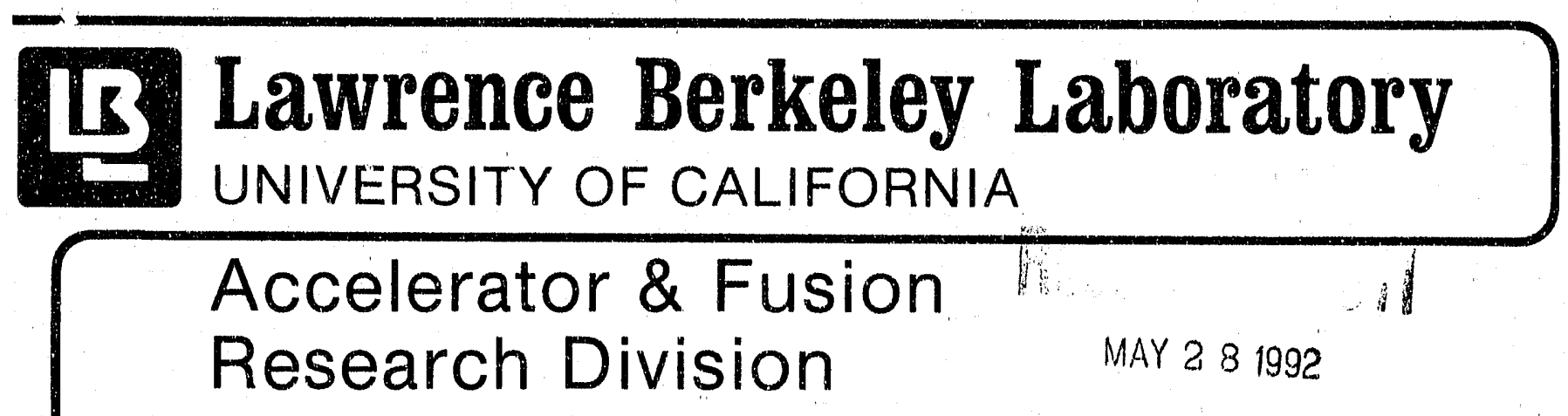

Presented at the Seventh National Conference on Synchrotron Radiation Instrumentation, Baton Rouge, LA, October 28-31, 1991, and to be published in the Proceedings

\title{
Scientific Program of the Advanced Light Source at LBL
}

\author{
A.L. Robinson and A.S. Schlachter
}

October 1991

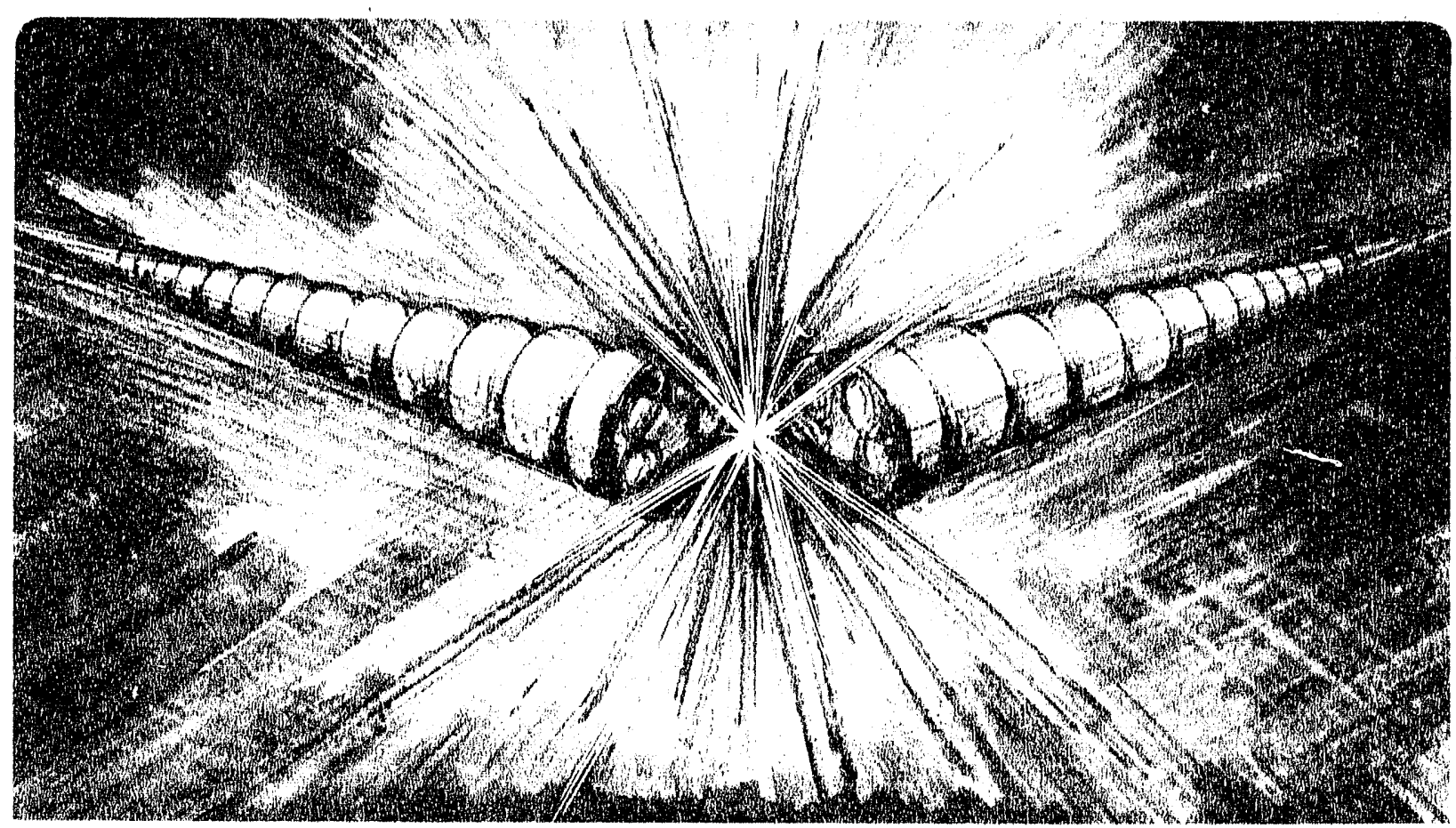

DISTRIBUTION OF THIS DOCUMENT IS UNLIMITEL

Prepared for the U.S. Department of Energy under Contract Number DE-AC03-76SF00098 


\section{DISCLAIMER}

This document was prepared as an account of work sponsored by the United States Government. Neither the United States Government nor any agency thereof, nor The Regents of the University of California, nor any of their employees, makes any warranty, express or implied, or assumes any legal liability or responsibility for the accuracy, completeness, or usefulness of any information, apparatus, product, or process disclosed, or represents that lis use would rot infringe privately owned rights. Reference herein to any specific iommercial product, process, or ser, ice by lts trade name, trademarl, manufacturer, or otherwise, does not necessarily constitute or imiply its endorsement, recommendation, or favoring by the United Btates Government or any agency thereof, or The Regents of the Uiniversity of California. The views and opinions of authors expressed herein do not necessarily state or reflect those of the United States Covernment or any agency thereof or The Regents of the University of California and shall not be used for advertising or product endorsement purposes.

Lawren ${ }^{-e}$ Berkeley Laboratory is an equal opportunity employer. 


\title{
Scientific Program of the Advanced Light Source at L BL*
}

\author{
A.L. Robinson and A.S. Schlachter \\ Advanced Light Source \\ Accelerator and Fusion Research Division \\ Lawrence Berkeley Laboratory \\ University of California \\ Berkeley, CA 94720
}

October 1991

Paper presented at the 7th National Conference on Synchrotron Radiation Instrumentation, Baton Rouge, LA, October 28-31, 1991

"This work was supported by the Director, Office of Energy Research, Office of Basic Energy Sciences, Materials Sciences Division of the U.S. Department of Energy, under Contract No. DE-AC03-76SF00098 


\title{
SCientific Program of The AdVANCEd Light SourCe AT LBL
}

\author{
A. L. Robinson and A. S. Schlachter \\ Advanced Light Source \\ Lawrence Berkeley Laboratory \\ University of California \\ Berkeley, CA 94720
}

\begin{abstract}
Construction of the Advanced Light Source (ALS) at the Lawrence Berkeley Laboratory is nearing completion, with operation as a U.S. Department of Energy national user facility scheduled to begin in the spring of 1993. Based on a low-emittance, $1.5-\mathrm{GeV}$ electron storage ring with 10 long straight sections available for insertion devices and, initially, 24 bend-magnet ports, the ALS will be a third-generation source of soft x-ray and ultraviolet (collectively, the XUV) synchrotron radiation. Experimental facilities (insertion devices, beamlines, and end stations) will be developed and operated by participating research teams working with the ALS staff. The ability to exploit the high spectral brightness of the ALS was the main criterion for PRT selection. In the XUV spectral regions served by the ALS, a major benefit of high brightness will be the ability to achieve spatial resolution in the neighborhood of 200 angstroms in $\mathrm{x}$-ray microscopy and holography and in spatially resolved spectroscopy. Other beneficiaries of high brightness include very-highresolution spectroscopy, spectroscopy of dilute species, diffraction from very small samples, and time-resolved spectroscopy and diffraction.
\end{abstract}

\section{The AdVANTAges OF BRIghtNess}

By 1993, so-called third-generation synchrotron sources will be coming on line in the United States, Europe, and Asia [1]. High spectral brightness (flux per unit area of the source, per unit solid angle of the radiation cone, and per unit bandwidth) is the main feature of the new thirdgeneration synchrotron facilities. High brightness is characterized by a high flux of highly collimated radiation onto a small area on the surface. In the past, order-of-magnitude increases in brightness have led to qualitatively new developments in spectroscopic and structural studies of both gas-phase and condensed matter [2].

Spatial resolution follows directly from high brightness, which makes it is possible to focus a large number of photons onto a small area. Spatial resolution most directly benefits those in the life and physical sciences who hope to achieve enhanced spatial resolution down to distance scales of about $200 \AA$ in $x$-ray microscopy and spatially resolved XUV spectroscopy. An example of the latter arises from the study of solid surfaces, which are mostly heterogeneous, making interpretation of spectroscopic data obtained from illurninating the entire surface difficult. With spatial resolution, spectrai features could be directly associated with specific surface areas and structures. For example, one could, track the features of a photoemission spectrum, such as the chemical shifts for an element in different chernical states, as the focused spot scans across the surface.

Spectral resolution is a second major benefit of high brightness. High spectral resolution is achieved by narrowing the slits of a monochromator. Consequently, there is a tradeoff between resolution and measuring time: the narrower the slits, the fewer photons pass through, and the longer the measuring time. High brightness allows a much larger portion of the photon bean to be focused through the slits, thereby dramatically increasing the resolution at fixed measurement time 
or decreasing the measurement time at nixed resolution. Experiments with once impractically long measuring times, perhaps because of inherently weak signals, now become reasonable to contemplate.

Spatial coherence implies the ability to form interference patterns. The most direct application of spatial coherence is the use of Fresnel zone plates to focus VUV and soft $x$ rays. Focusing is necessary to achieve high spatial resolution in scanning systems with small spots. Other applications include $x$-ray holography, a technique with the promise of providing the threedimensional images of biological and other structures.

Time resolution is a feature of all synchrotron sources that follows from the bunched character of the beam in the storage ring. The naturally pulsed nature of synchrotron radiation can be used to observe short-lived or transient systems by means of time-resolved spectroscopic, scattering, and imaging experiments. For example, if radiation damage due to the $x$-ray exposure can be avoided or minimized, it may be possible to image changes in functioning biological cells and cellular structures in near-natural environments. The ultimate time resolution, made possible if there are enough photons in a single pulse of bright synchrotron light to generate a useful signal, would be to follow events in real time on the sub-nanosecond time scale. Time resolution can also be achieved by operating in synchrony with other light sources, such as a high-speed laser in a pumpprobe mode.

Polarization control is another feature that has always been associated with synchrotron sources. With bend magnets, radiation is largely linearly polarized when viewed in the plane of the electron orbit, but it is elliptically polarized when viewed at angles above or below the plane. With special insertion devices, undulators and wigglers, it is also possible to generate elliptically polarized beams, in particular, circularly polarized beams, which can be used to investigate structures with a handedness (chiral structures) and magnetic materials. New insertion-device and beam-line technology, in combination with high-brightness synchrotron sources, promises to bring the now specialized uses of controlled polarization into the mainstream of synchrotron research.

High flux is associated with high brightness, although high brightness is not necessary for high flux. Many experiments are primarily flux limited: The number of atoms or molecules, such as impurities in a semiconductor or ionized atoms in a vapor, may be too small to generate a measurable signal in a practical time. A high photon flux effectively compensates for the small number of signal generators by exciting them more often, thereby making impractical experiments feasible.

\section{The Advanced Light SOURCE}

The Advanced Light Source, a next-generation syrichrotron source of XUV radiation, is under construction in Berkeley, California, at the Lawrence Berkeley Laboratory (LBL) of the University of California (Fig. 1). The ALS is a U.S. Department of Energy-funded construction project with a construction cost of $\$ 99.5$ million. The project is scheduled to be completed in April 1993. As a national user facility, the ALS will be available to visiting and in-house researchers from university, industrial, and federal laboratories.

The ALS facility consists of an accelerator complex, a complement of insertion devices, beamlines, and associated experimental apparatus, and a building to house this equipment. The status of the construction project is reviewed in the paper by Marx [3]. The accelerator complex comprises a $50-\mathrm{MeV}$ linac, a $1-\mathrm{Hz}, 1.5-\mathrm{GeV}$ booster synchrotron, and an electron storage ring. Table 1 lists the major parameters of the storage ring [4]. The storage ring is optimized for the use of insertion devices. Of the 12 straight sections in the storage ring, one is used for injection and one is occupied by if cavities, leaving 10 full straight sections available for undulators and 
wigglers up to $4.5 \mathrm{~m}$ in length. Each arc of the storage ring is fitted with four bend-magnet ports. Of the maximum of 48 ports, 24 are so-called prime ports with superior properties and will be developed first.

In the normal storage-ring operating mode (the multibunch mode with a 400 -mA current), the time structure of the radiation will comprise pulses with a full-width-half-maximum (FWHM) of about 35 ps and separation of 2 ns. For time-resolved experiments, a few-bunch mode will be available in which the nominal current per bunch will be $7.6 \mathrm{~mA}$ and the bunch length is predicted to be $55 \mathrm{ps}$, although still larger bunch currents may be tolerated (with additional bunch lengthening) before the beam becomes unstable

\section{INSERTION-DEVICE RADIATION SOURCES}

The magnetic structure of an insertion device consists of a linear array of north-south dipoles of alternating polarity. The normal vertical orientation of the dipoles causes relativistic electrons of energy $E$ to undergo a nearly sinusoidal electron trajectory of period $\lambda_{u}$ in the horizontal plane, causing the emission of synchrotron radiation, as shown in Fig. 2.

The value of deflection parameter $K$ is used to distinguish between an undulator and a wiggler, where $K$ is the ratio of the maximum angular deviation of the electron trajectory from the insertiondevice axis to the natural opening angle of the synchrotron-radiation cone. Expressed in terms of the undulator peak magnetic field $B_{0}$ and period $\lambda_{\mu}, K$ is approximately

$$
K=0.934 B_{0}[\text { Tesla }] \lambda_{l l}[\mathrm{~cm}]
$$

The value of $K$ at the breakpoint between an undulator and a wiggler is somewhat arbitrary. Most of the undulators planned for the ALS operate in an interrnediate range with $K<1$ at one end and $K>1$ at the other. When $K \gg 1$, the structure is called a wiggler.

In the undulator regime, the radiation emitted from successive undulator periods adds coherently. The interference behavior due to the coherence gives rise to a sharply peaked radiation spectrum consisting of a fundamental and several harmonics. The photon energy, $\varepsilon_{n}$, of the $n$th harmonic is

$$
\varepsilon_{n}[\mathrm{keV}]=\frac{0.949 n E^{2}[\mathrm{GeV}]}{\lambda_{u}[\mathrm{~cm}]}\left(\frac{1}{1+K^{2} / 2+\gamma^{2} \theta^{2}}\right)
$$

where $\gamma$ is ratio of the electron relativistic and rest masses and $\theta$ is the angle of emission relative to the undulator axis. At the ALS, use of the third and fifth harmonics of the undulators is planned to extend their spectral range to higher photon energies than can be reached with the fundamental alone.

By contrast, the lack of in erference in a wiggler means that its synchrotron radiation spectrum is like the broad, continuous spectrum from the dipole or bend magnets in the curved sections of the storage ring. The wiggler spectrum is characterized by a critical photon energy $\varepsilon_{C}$, defined as the photon energy above which half the total power is radiated. At the high end of the broad wiggler spectrum, the flux drops rapidly but is still one-tenth of its maximum value at photon energies near $4 \mathrm{e}_{C}$ 
Operating at $1.5 \mathrm{GeV}$, the ALS is optimized for insertion-device operation in the XUV spectral regions. Between them, the undulators will be able to excite the $\mathrm{K}$ shell of elements through silicon and the $\mathrm{L}$ shell of elements up to krypton, while the wiggler will be able to excite the L shell of nearly every element in the periodic table. Table 2 lists the properties of three undulators (U8.0, U5.0, and U3.9, where the number refers to the period length in centimeters) that span the XUV spectral region when the ALS operates at $1.5 \mathrm{GeV}$ [5]. In addition, undulator U10, 0 , has been requested by a user group specifically to provide low-energy photons in the range 6-50 eV. With an $\varepsilon_{C}$ of $3.1 \mathrm{keV}$, the wiggler in Table 2 extends the ALS spectral range into the hard $x$-ray region above $10 \mathrm{keV}$. High-quality synchrotron radiation will be available from the 24 prime bendmagnet ports as well. The critical photon energy of the bend magnets is $1.56 \mathrm{keV}$. Figure 3 shows the spectral brightness of the ALS radiation sources along with that of representative sources at other facilities.

A significant fraction of the radiation from the ALS undulators is spatially coherent. The criterion for spatial coherence is that the product of the area of the light source and the solid angle into which it emits be no larger than the square of the wavelength of the light. Since this is the diffraction condition, spatially coherent light is also said to be diffraction-limited. In accordance with the diffraction condition, the electron-beam emittance $\varepsilon$ sets the minimum wavelength at which all the radiation can be diffraction limited, according to the relation

$$
\varepsilon=\lambda_{\min } / 4 \pi
$$

Even at wavelengths below the minimum, part of the radiation remains diffraction limited, the fraction decreasing as the square of the wavelength.

Although phase-sensitive techniques, such as holography, most naturally come to mind when thinking about coherent radiation, a more general virtue is the ability to focus. For example, a Fresnel zone plate can focus a coherent beam of soft $x$-rays to a spot whose radius is approximately 1.2 times the width of the outermost zone. With state-of-the-art microfabrication techniques, such as electron-beam holography, it is possible to make zone plates with outer zone widths of about $300 \AA$ (Fig. 4). This capability can be exploited in scanning or imaging systems to generate spatially resolved information with a comparable resolution.

\section{SCIENTific Program}

The ALS capabilities will support an extensive research program in a broad spectrum of scientific and technological areas in which $\mathrm{x}$-ray and ultraviolet radiation is used to study and manipulate matter in all its varied gaseous, liquid, and solid forms. New opportunities for research in materials, interface, and surface science, in atomic and molecular physics, in chemistry, in the life sciences, in earth science, and in X-ray optics will be available. The ALS will also serve those interested in developing the fabrication technology for micro- and nanostructures, as well as for characterizing them.

The initial scientific program emphasizes the high brightness of XUV light available from the ALS. The program is being implemented by means of participating research teams (PRTs) consisting of investigators with related research interests from one or more institutions. The primary responsibility for experimental apparatus rests with the PRTs; the responsibility for the beamlines and, where appropriate, insertion devices will be shared between the ALS and the PRTs. In return for its commitment, each PRT receives a guaranteed fraction of the ALS operating time at its beamline. A substantial fraction of the ALS running time at each beamline will also be made available to independent investigators not affiliated with a PRT by means of a proposal 
process.

PRTs working with undulator or wiggler beamlines are called insertion-device teams. Other PRTs work with bend-magnet beamlines and are known as bend-magnet teams. At present there are eight approved insertion-device teams and eight approved bend-magnet teams. Figure 5 shows a layout of the ALS building with possible locations of insertion devices and associated beamlines for the approved teams. Implementation of the scientific program relies heavily on the success of the PRTs in obtaining funding for their share of the experimental facilities that they propose. As of November 1991, three insertion-device and two bend-magnet PRTs have succeeded and will be ready to take data when the ALS begins operations. Two other bend-magnet teams are likely to be on the floor, as well. These groups include:

- U5.0 undulator. An insertion-device team with Brian Tonner of the University of Wisconsin-Milwaukee and the University of Wisconsin-Madison Synchrotron Radiation Center as spokesperson will operate a "Spectromicroscopy Facility" with multiple end stations that emphasizes spatially resolved photon and electron spectroscopic techniques, including photoelectron microscopy, diffraction and holography, applied primarily to materials, surfaces, and interfaces in the photon-energy range from 80 to $1100 \mathrm{eV}$..

- U5.0 undulator. An insertion-device team with Joachim Stöhr of the IBM Almaden Research Center, Franz Himpsel of the IBM T.J. Watson Research Center, Thomas Callcott of the University of Tennessee and David Ederer of the National Institute of Standards and Technology as joint spokespersons will operate a high-resolution core-level spectroscopy beamline with multiple end stations for photon and electron spectroscopy of materials, surfaces, and interfaces in the photon-energy range from 90 to $1500 \mathrm{eV}$; the beamine will include spectromicroscopy and fluorescence spectroscopy capabilities.

- U8 undulator. An insertion-device team with Denise Caldwell of the University of Central Florida as the spokesperson will operate a beamline for photoelectron, photoionization, and fluorescence spectroscopy of atoms, molecules, and ions at photon energies from $20-300 \mathrm{eV}$; the beamline will have separate branches for investigation of ionic systems and for study of actinide materials.

- Bend magnet. A bend-magnet team with Charles Fadley of the University of California at Davis and the Lawrence Berkeley Laboratory as the spokesperson will operate a VUV-Soft $\mathrm{x}$-ray beamline for high-resolution photon, photoelectron (including photoelectron diffraction and holography), and photoionization spectroscopy of materials and chemical systems at photon energies from 30 to $1250 \mathrm{eV}$.

- Bend magnet. A bend-magnet team with Albert Thompson of the Lawrence Berkeley Laboratory as the spokesperson will operate an X-ray fluorescence microprobe beamline for nondestructive elemental analysis of materials in ambient environments with a spatial resolution of $1 \mu \mathrm{m}^{2}$ and elemental sensitivity in the femtogram range; the $\mathrm{x}$-ray excitation will be tunable over the photon-energy range from 1 to $12 \mathrm{keV}$ to maximize elemental sensitivity.

- Bend magnet. A bend-magnet te $m$ with Dennis Lindle of the University of Nevada at Las Vegas as spokesperson proposes to operate a beamline emphasizing photon absorption and emission, photoelectron, photoionization, and photodissociation spectroscopy for atomic, molecular and optical physics over the photon-energy range from $500 \mathrm{eV}$ to $5 \mathrm{keV}$; additional funding is needed to implement this proposal.

- Bend magnet. A bend-magnet team with Marvin Weber of the Lawrence Livermore National Laboratory as spokesperson proposes a VUV-soft $\mathrm{x}$-ray beamline for high-resolution photon 
and electron spectroscopy (including photoelectron diffraction and holography) of materials and chemical systems at photon energies from 60 to $1100 \mathrm{eV}$ and for testing of optical components and instrumentation; the beamline will have a circular polarization capability; additional funding is needed to implement this proposal.

These beamlines collectively represent a substantial initial investment in experimental facilities with the capability of producing much exciting, forefront science. It is possible that there will be additional insertion-device and bend-magnet beamlines for biological x-ray microscopy and and for advanced projection $x$-ray lithography development within a short time. In the long run, a steady growth toward the full ALS experimental program is envisioned for future years.

\section{REFERENCES}

[1] Barbara Goss Levi, Phys. Today 44, 17 (April 1991).

[2] Herman Winick, Sci. Am. 257, 88 (November 1987).

[3] Jay N. Marx, [this proceedings].

[4] 1-2 GeV Synchrotron Radiation Source, Lawrence Berkeley Laboratory PUB-5172 Rev, 1986 (unpublished). Note that the values of some ALS parameters have changed since the issuance of this report.

[5] An ALS Handbook, Lawrence Berkeley Laboratory PUB-643 Rev. 2, 1989 (unpublished).

[6] W. Meyer-Ilse et al., "Experimental Characterization of Zone Plates for High Resolution XRay Microscopy," in X-ray Microscopy III, A. Michette et al. eds., Berlin, Springer-Verlag (in press). 
Table 1 Main Parameters of the ALS Storage Ring

\begin{tabular}{cl}
\hline Beam energy [GeV] & 1.5 \\
Nominal & 1.0 \\
Minimum & 1.9 \\
Maximum & 196.8 \\
Circumference [m] & 400 \\
Beam current [mA] & 7.6 \\
Multibunch & $<10^{-8}$ \\
Single bunch & $<10^{-9}$ \\
Beam emittance, rms [m.rad] & 6 \\
Horizontal & $30-50$ \\
Vertical & 12 \\
Beam lifetime [h] & 10 \\
Nominal bunch duration, FWHM [ps] & 4.5 \\
Number of straight sections & \\
Number available for insertion devices &
\end{tabular}


Table 2. Parameters for ALS Insertion Devices

\begin{tabular}{|c|c|c|c|c|}
\hline Name & $\begin{array}{l}\text { Period } \\
{[\mathrm{cm}]}\end{array}$ & $\begin{array}{l}\text { No. of } \\
\text { Periods }\end{array}$ & $\begin{array}{l}\text { Photon Energy } \\
\text { Range }[\mathrm{eV}] \mathrm{a})\end{array}$ & $\begin{array}{l}\text { Critical } \\
\text { Energy [keV] }\end{array}$ \\
\hline Undulators. & & & & \\
\hline U8.0 & 8.0 & 55 & $\begin{array}{l}5.4-220 \mathrm{~b}) \\
{[16.2-660]} \\
{[27-1100]}\end{array}$ & - \\
\hline U5.0 & 5.0 & 89 & $\begin{array}{c}52-380 \\
{[156-1140]} \\
{[260-1900]}\end{array}$ & - \\
\hline U3.9 & 3.9 & 115 & $\begin{array}{c}169-500 \\
{[507-1500]} \\
{[845-2500]}\end{array}$ & - \\
\hline $\begin{array}{l}\text { Wiggler } \\
\text { W16 }\end{array}$ & 16 & 16 & - & 3.1 \\
\hline
\end{tabular}




\section{FIGURE CAPTIONS}

Fig. 1. The ALS building at the Lawrence Berkeley Laboratory comprises two parts: the inner section under the dome (1900 square-meter area) houses a linear accelerator and a booster synchrotron; the annular section (5700 square-meter area) shelters the storage ring and the experimental areas. (CBB 913-1534 cropped)

Fig. 2. Diagram of the magnetic structure of an undulator shows the main features of the hybrid technology that uses permanent-magnet material to drive steel pole pieces. North-south dipoles oi alternating polarity bend electrons moving at nearly the speed of light into a sinusoidal trajectory, thereby causing the emission of a narrow cone of XUV radiation of high spectral brightness. (XBL $831-7589 \mathrm{E})$

Fig. 3. With the use of more than one undulator harmonic it is possible to cover a wide spectral range. The spectral brightness envelopes are shown for the three ALS undulators and wiggler described in Table 2, the ALS bend magnets, undulators planned for the Advanced Photon Source (APS), and representative insertion-device and bend-magnet sources at the National Synchrotron Light Source (NSLS) and the Stanford Synchrotron Radiation Center (SSRL). X-1 is an undulator beamline on the NSLS $x$-ray ring. The discontinuities in the undulator curves represent a shift from the envelope of the fundamental to that of the third harmonic, etc. (XBL 893-7098A)

Fig. 4. Details in this $x$-ray micrograph of a gold test pattern with 128 radial spokes on a silicon nitride substrate are well resolved to $360 \AA$, and some details as fine as $300 \AA$ can be seen with reduced contrast. The micrograph was made with the use of a Fresnel zone plate $x$-ray lens with an outer zone width of $300 \AA$ in an imaging $x$-ray microscope with 24 - $\AA$ x-rays [6]. (XBB 9043182A)

Fig. 5. Fioor plan showing a possible placement of insertion devices and locations of associated beamlines in the ALS building. The maximum beamline length is about $35 \mathrm{~m}$. The prime bendmagnet ports to be developed initially are located in the center bend magnet of each storage-ring arc. At two ports per magnet, there is a total of 24 ports. (XBL 915-955) 


\section{DISCLAIMER}

This document was prepared as an account of work sponsored by the United States Government. Neithe: the United States Government nor any agency thereof, nor The Regents of the University of California, nor any of their employees, makes any warranty, express or implied, or assumes any legal liability or responsibility for the accuracy, completeness, or usefulness of any inforination, appara' $2 \mathrm{~s}$, product, or process disclosed, or represents that its use would not infringe privately owned rights. Reference herein to any specific commercial product, process, or service by its trade name, trademark, manufacturer, or otherwise, does not :ecessarily constitute ur imply its endorsement, recommendation, or favoring by the United States Government or any agency thereof, or The Regents of the University of Caltfornia. The views and opinions of authors expressed herein do not necessarily state or reflect those of the United States Government or any agency thereof or The Regents of the University of Cilifornia and shall not be used for advertising or product endorsement purposes.

Lawrence Berkeley Laboratory is an equal opportunity employer. 

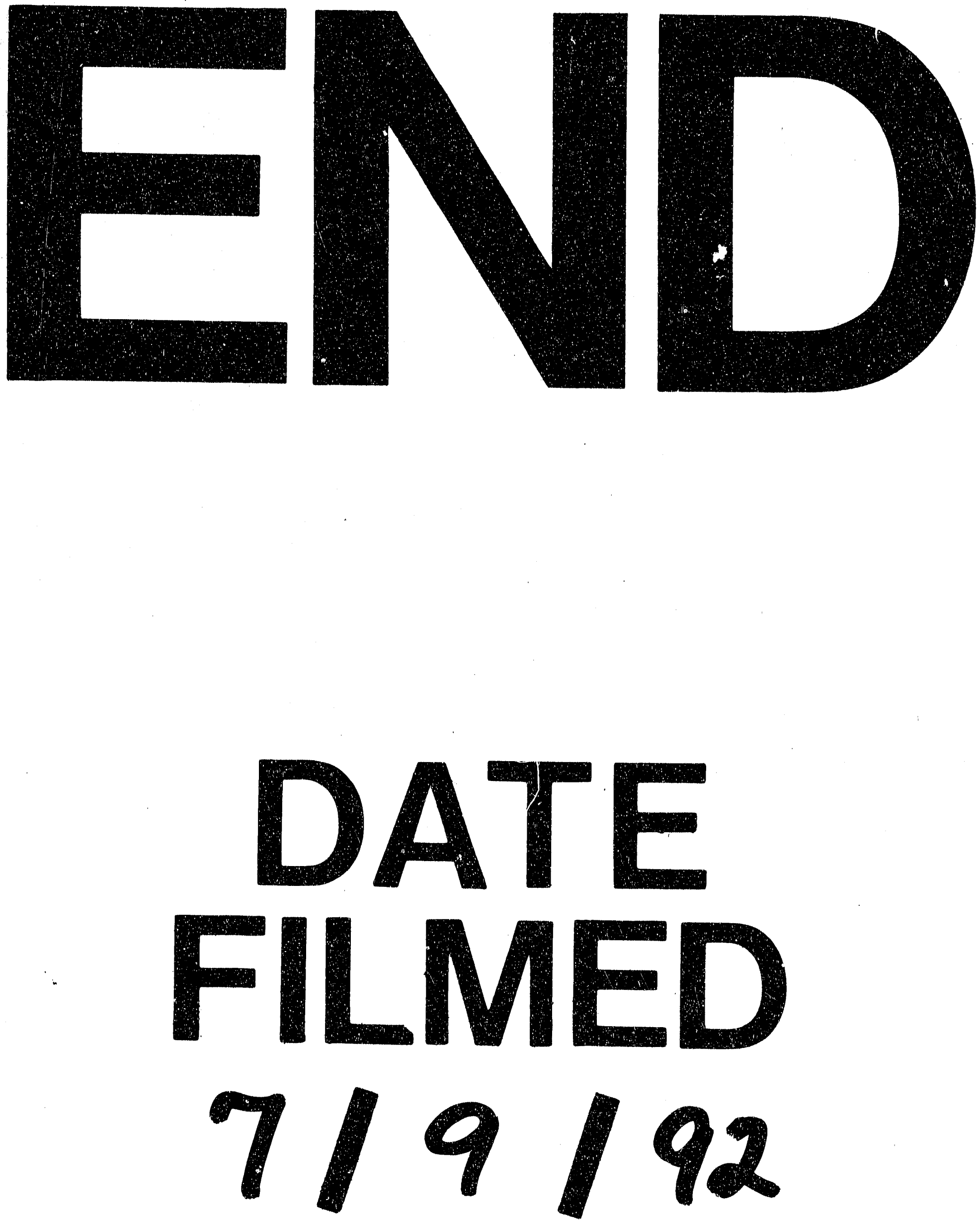
